\title{
O PARTO HORIZONTAL E O RISCO DE LESÕES MÚSCULO- ESQUELÉTICAS NOS ENFERMEIROS OBSTETRAS
}

\author{
Armando Sousa ${ }^{1,3}$; Maria Helena Presado ${ }^{2,3}$ \\ ${ }^{1}$ Escola Superior de Enfermagem São José Cluny no Funchal, Portugal. armandodav@gmail.com \\ 2 Escola Superior de Enfermagem de Lisboa, Portugal \\ 3 Unidade de Investigação e Desenvolvimento em Enfermagem, da ESEL de Lisboa, Portugal. \\ mhpresado@esel.pt
}

\begin{abstract}
Resumo. A exigência física, o ambiente inadequado, o stress inerente e a carga emocional subjacente, são alguns dos fatores que favorecem o aparecimento das lesões músculoesqueléticas ligadas ao trabalho (LMELT). Este fenómeno tem sido grandemente analisado pela comunidade científica no núcleo da enfermagem, não contemplando, no entanto, os enfermeiros obstetras. Objetivos: Os objetivos são analisar os fatores que dificultam a adoção dos princípios da biomecânica, pelo enfermeiro obstetra, e identificar o nível de risco para LMELT, das atividades desenvolvidas durante o segundo e terceiro estádio do parto horizontal. Métodos: Esta investigação do tipo descritiva e exploratória, com metodologia mista, de interação independente e convergindo na interpretação dos resultados, tem como questão: quais as posturas adotadas pelo enfermeiro obstetra, na assistência à parturiente durante o segundo e terceiro estádios do trabalho de parto em posição horizontal, que favorecem o aparecimento das LMELT? Resultados: Foi identificado alto risco de LMELT em 11 atividades realizadas pelos enfermeiros obstetras, e risco muito elevado em 2 atividades. Verificámos que a posição predominante foi flexão da cabeça, elevação dos ombros, e tronco desalinhado e em flexão. Conclusão: Este estudo permitiu a análise ergonómica dos enfermeiros obstetras na sua praxis, para posterior intervenção-ação.
\end{abstract}

Palavras-chave: Lesões Músculo-Esqueléticas; enfermeiro obstetra; análise ergonômica; partos horizontais; segundo e terceiro estádios do trabalho de parto.

\section{HORIZONTAL DELIVERY AND THE RISK OF MUSCULOSKELETAL INJURIES IN OBSTETRIC NURSE}

Abstract. The physical demands, the inadequate environment, the inherent stress and the underlying emotional load are some of the factors that favor the appearance of Work related musculoskeletal disorde (WRMSD). This phenomenon has been highly analyzed by the scientific community in the nursing nucleus, but obstetric nurses were not considered. Goals: The objectives are to analyze the factors that hinder the adoption of the principles of biomechanics by the obstetric nurse and to identify the level of risk, for WRMSD in activities developed during the second and third stage in horizontal deliveries. Methods: This descriptive and exploratory investigation with mixed methodology, of independent interaction and converging in the interpretation of the results, has the following question: what are the postures adopted by the obstetric nurse, in assisting the parturient during the second and third stages of labor in a horizontal position, which favor the appearance of WRMSD?. Results: A high risk of WRMSD was identified in 11 activities performed by obstetric nurses and a very high risk in 2 activities. We found that the predominant position was flexion of the head, elevated shoulders and misalignment and flexion of the trunk. Conclusion: This study allowed the ergonomic analysis of obstetric nurses in their practice, for later interventionaction

Keywords: Musculoskeletal Disorders; Obstetric Nurse; Ergonomic Postures; Horizontal Delivery Labor Stage; Second and Third.

\section{INTRODUÇÃO}

As LMELT são doenças ocupacionais causadas por traumas cumulativos, resultantes da descompensação entre a capacidade funcional do músculo e a sua execução e frequência 
(Wang, Cui, Xu, Yuan, Jin \& Li, 2017). O desequilíbrio entre as solicitações mecânicas do trabalho, a capacidade de adaptação de uma região corporal e a insuficiência de períodos de pausa/recuperação, têm como possível consequência as LMELT (Jerónimo, 2013).

São várias as estruturas anatómicas que podem ser atingidas, incluindo musculatura, articulações, tendões, ligamentos, nervos, estruturas ósseas, bem como o aparelho circulatório, sendo a região cervical, ombros, membros superiores e a região lombar as áreas corporais mais comumente afetadas (AESST, 2007). Incluem-se, na sintomatologia apresentada por estes profissionais: dor, fadiga ou desconforto localizado, parestesias na região afetada ou em áreas subjacentes, a sensação de peso, sensação ou perda objetiva de força muscular, e o edema (Queiroz, Uva, Carnide, Serranheira, Miranda, \& Lopes, 2008). O surgimento desta sintomatologia é gradual, acentuando-se no final do dia de trabalho ou durante os picos de produção, aliviando com o repouso e descanso (Jerónimo, 2013).

A interligação e eficiência entre o sistema músculo-esquelético e nervoso, que sustenta o equilíbrio, a postura e o alinhamento do corpo durante as diferentes atividades de vida do indivíduo, designa-se por mecânica corporal, comportando cinco princípios: o alinhamento corporal, o equilíbrio corporal, o movimento, a fricção/atrito e a força mecânica (OE, 2013; Costa et al., 2011).

A realização do parto de baixo risco é uma das competências autónomas do enfermeiro obstetra que compreende uma atenção especial dada a sua especificidade e componente emocional subjacente (Long, Bogossian \& Johnston, 2013).

A ocorrência da dilatação completa $(10 \mathrm{~cm})$ e descida da apresentação fetal pelo pavimento pélvico, denomina-se segundo estádio do trabalho de parto. O terceiro estádio começa com a saída da apresentação e termina com a expulsão da placenta (Fatia \& Tinoco, 2016).

Segundo a Agência Europeia de Segurança e Saúde no Trabalho (AESST, 2007), o problema de saúde ocupacional mais frequente na Europa são as lesões músculoesqueléticas, com uma prevalência de $24 \%$ de lombalgias e de $22 \%$ de dores musculares (EU-25), com tendência a aumentar a sua incidência. As consequências a nível pessoal são o sofrimento, a perda de rendimento e o aumento do risco de cronicidade, enquanto que, a nível empresarial, verifica-se a redução da produtividade, o aumento do absentismo, o compromisso da capacidade produtiva e aumento dos custos (Hoof et al., 2018). 
A comunidade científica atual tem manifestado um enorme interesse no estudo das LMELT nos enfermeiros, bem patente no elevado número de estudos realizados com este grupo profissional. (Davis \& Kotowski, 2015). Os estudos evidenciam uma elevada prevalência de LMELT nestes profissionais, com valores entre 71,85\% a 84\% (Akbari, Akbari, Abadi, Fesharaki \& Ghaseni, 2017; Tsekoura, Koufogianni, Billis, \& Tsepis, 2017; Ellapen \& Narsigan, 2014).

As áreas do organismo mais atingidas são a região lombar, cervical, os ombros, joelhos e região torácica. A prevalência nestas regiões, varia de acordo com o país; a América do Norte, em geral, apresentou as taxas mais baixas, o Médio-oriente, a Ásia, a Austrália e Filipinas foram os países com maior prevalência de LME em todas as regiões corporais (Davis \& Kotowski, 2015).

Chung, et al. (2013), verificou que os problemas documentados de LMELT nos enfermeiros são: patologias de coluna (incluindo hérnias discais), síndrome do ombro doloroso, epicondilites mediais, dedo em gatilho e síndrome do canal cárpico.

Em Portugal os estudos apenas fazem referência à elevada prevalência de LMELT em serviços como a Medicina, Ortopedia, Cirurgia e Reabilitação (Santos, 2015; Jerónimo, 2013).

Não foram encontrados estudos de prevalência das LMELT nos enfermeiros obstetras (EO) a nível nacional, mas o estudo de autoperceção dos fatores de risco realizado por Prezado et al. (2015), evidenciou que a maioria dos EO já haviam sofrido uma LMELT, com incapacidade temporária para o trabalho. Num estudo chinês realizado ao staff do serviço de Ginecologia e Obstetrícia, conclui-se que a prevalência de LMELT para este grupo era de $85,5 \%$ (Wang et al., 2017).

\subsection{Objetivos/Questões}

$\mathrm{Na}$ abordagem quantitativa pretende-se identificar o nível de risco para LMELT do enfermeiro obstetra, durante o segundo e terceiro estádio do trabalho de parto, em partos horizontais. Relativamente à abordagem qualitativa, queremos analisar os princípios da biomecânica adotados pelos enfermeiros obstetras na assistência à parturiente, durante o segundo e terceiro estádio do trabalho de parto, em parto horizontal. Pretendemos ainda identificar os fatores que dificultam a adoção dos princípios da biomecânica. 
A questão do estudo foi: Quais as posturas adotadas pelo enfermeiro obstetra, na assistência à parturiente durante o segundo e terceiro estádio do trabalho de parto em posição horizontal, que favorecem o aparecimento de LMELT?

\subsection{População, contexto e amostra}

A população do estudo foram os enfermeiros obstetras de qualquer idade, género, cultura e raça, que desempenham a sua atividade profissional em contexto hospitalar, especificamente no bloco de partos, e que assistiram às parturientes em partos horizontais, no decorrer do segundo e terceiro estádios do trabalho de parto, durante o mês de janeiro de 2018. O tipo de amostragem foi não probabilístico ou não-aleatório, dado que, não seguiu os princípios básicos da teoria das probabilidades, e foi uma amostragem intencional de especialistas, porque os elementos possuem conhecimentos/competências específicos na abordagem ao parto eutócico.

\section{METODOLOGIA}

Na fase empírica de colheita de dados (operacionalização da recolha, organização e análise dos dados), foram utilizadas 50 videogravações, decorrentes de 25 partos (25 vídeos laterais e 25 vídeos posteriores), do segundo e terceiro estádio do trabalho de parto, recorrendo a duas câmaras.

Para a análise dos vídeos foi utilizado o método proposto por Lima (2015), que contempla seis fases: visualizar os vídeos, selecionar os eventos críticos; descrever os eventos críticos, transcrever os eventos críticos, discutir os dados encontrados e eliminar os vídeos (Lima, 2015).

Após a visualização completa de todos os vídeos, foram selecionados os eventos críticos em que o fenómeno é expresso e tem ação (Powell, Francisco \& Maher, 2004). Foram narrados sobre a forma de texto, transcritos para o software WebQdA®, posteriormente codificados, categorizados e interpretados (Flick, 2004).

Para a análise das posturas adotadas pelo enfermeiro obstetra, no decorrer dos eventos críticos, foi utilizada uma grelha de observação que contempla os indicadores referentes aos princípios da biomecânica, adotada por outros investigadores (Baixinho et al., 2017). Foi ainda aplicada a escala REBA, que permite avaliar o risco de LMELT e a prioridade de atuação. 
Foram identificadas 21 atividades de risco, que corresponderam a 21 frames, sujeitos à técnica de análise postural lateral e posterior pelo REBA (Rapid Entire Boby Assessment) de Hignett e McAtamney (2000). Esta técnica contempla a divisão do corpo humano em seis segmentos distintos: pescoço, tronco, pernas, braços, antebraços e punhos. Foi necessário calcular o nível de risco para cada segmento do corpo e, posteriormente, efetuar a correspondência de ambos os segmentos numa tabela específica, obtendo a pontuação final do REBA.

$\mathrm{Na}$ análise e interpretação qualitativa dos vídeos, foi adotada a técnica proposta por Bardin (2013), recorrendo às três fases em todo este processo: a pré-análise, onde o quadro de referência teórico foi elaborado, analisado e utilizado para estabelecer os indicadores, mantendo a exaustividade, a representatividade, homogeneidade e pertinência dos dados; exploração do material, onde foram construídas as operações de codificação, tendo por base as unidades de registo do texto, definindo regras de contagem, classificação e agregação da informação em categorias simbólicas ou temáticas; por fim, o tratamento dos resultados, inferência e interpretação (Silva \& Fossá, 2015; Bardin, 2013).

O recurso ao software webQDA ${ }^{\circledR}$ e a atribuição de um código de cores, facilitou a codificação e a definição das categorias de forma estruturada e interligada.

$\mathrm{Na}$ elaboração do código de árvore relativamente aos fatores de risco físicos e organizacionais/psicossociais, foi tida em consideração a grelha de categorização elaborada por Baixinho et al., (2017), relativamente aos princípios da biomecânica (movimento corporal, alinhamento corporal, equilíbrio, força, atrito e fricção).

No processo de categorização, emergiram três categorias relacionadas com o risco físico: mecânica corporal, ergonomia do bloco de partos e relativa à atividade (figura 1); e uma categoria relativa aos fatores de risco psicossociais (autonomia na orientação do parto). 


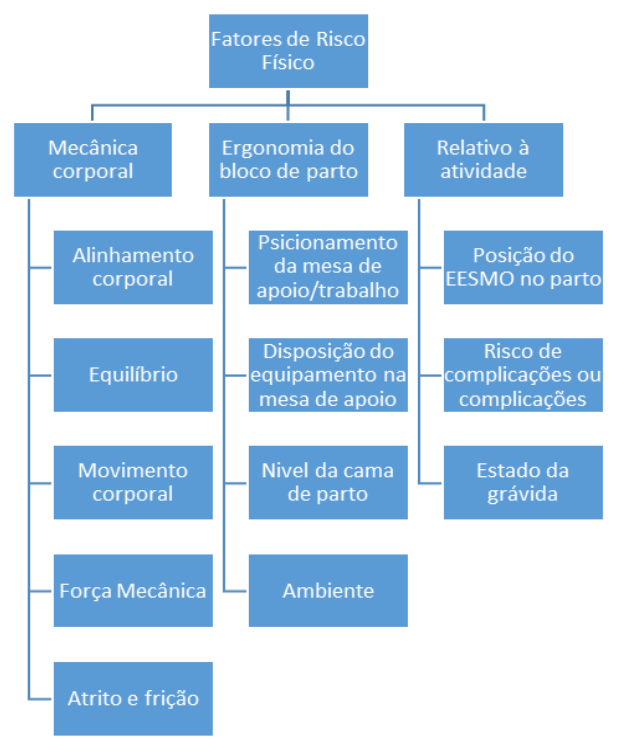

Figura 1. Esquema de categorização relativamente aos fatores de risco físico

No decorrer deste estudo foram assegurados os princípios éticos, englobando autodeterminação, intimidade, anonimato e confidencialidade, proteção contra o desconforto e prejuízo, tratamento justo e equitativo. Foi elaborado um consentimento livre e esclarecido para todos os intervenientes (enfermeiro obstetra, grávida, companheiro e outros profissionais de saúde) e obtida a autorização por parte do Conselho de Administração e da Comissão de Ética para a realização do estudo, parecer nํ. 44/2017 de 14/11/217).

Para proceder à gravação do parto, foi realizada uma primeira abordagem ao casal, aquando da sua admissão no bloco de partos, com explicação e esclarecimento de dúvidas. O casal disponha de um período de reflexão até à dilatação completa e, caso concordasse em participar, era efetuada a assinatura do consentimento informado. Antes do início da gravação, a autorização dos intervenientes era confirmada verbalmente.

\section{RESULTADOS}

As atividades que obtiveram maior score de risco (risco muito elevado), foram as atividades $11(11,3)$ e $8(10,4)$, que correspondem à "expressão facial do recém-nascido para libertação de secreções" e a "libertação do ombro anterior", respetivamente. Das restantes 19 atividades, onze obtiveram uma avaliação de alto risco para LMELT.

A análise dos vídeos (tabela 1), permite-nos verificar que, das 902 unidades de enumeração (FI) obtidas, 887 unidades correspondem aos fatores de risco físico e apenas 15 aos fatores psicossociais e organizacionais. 
Tabela 1 - Esquema de categorização relativamente aos fatores de risco físico

\begin{tabular}{|c|c|c|c|}
\hline Fator de Risco & Categoria & Subcategoria & $E U$ \\
\hline \multirow{4}{*}{ Físico } & $\begin{array}{l}\text { Mecânica } \\
\text { Corporal }\end{array}$ & $\begin{array}{l}\text { Alinhamento corporal } \\
\text { Equilíbrio } \\
\text { Movimento corporal } \\
\text { Fricção e atrito } \\
\text { Força mecânica } \\
\text { Subtotal }\end{array}$ & $\begin{array}{c}184 \\
189 \\
151 \\
75 \\
125 \\
724\end{array}$ \\
\hline & $\begin{array}{l}\text { Relativo à } \\
\text { atividade/taref } \\
\text { a }\end{array}$ & $\begin{array}{l}\text { Posição do enfermeiro obstetra } \\
\text { Risco de complicações ou complicações } \\
\text { Estado da grávida } \\
\text { Subtotal }\end{array}$ & $\begin{array}{l}26 \\
14 \\
25 \\
65\end{array}$ \\
\hline & $\begin{array}{ll}\text { Ergonomia } & \text { do } \\
\text { bloco } & \text { de } \\
\text { partos } & \end{array}$ & $\begin{array}{l}\text { Posicionamento da mesa de apoio/trabalho } \\
\text { Disposição do equipamento /material na mesa } \\
\text { Nível da cama de parto } \\
\text { Ambiente } \\
\text { Subtotal }\end{array}$ & $\begin{array}{l}25 \\
14 \\
26 \\
33 \\
98\end{array}$ \\
\hline & \multirow{2}{*}{\multicolumn{2}{|c|}{$\begin{array}{c}\text { Total } \\
\text { Autonomia na orientação do parto }\end{array}$}} & 887 \\
\hline Psicossociais e organizacionais & & & 15 \\
\hline \multicolumn{3}{|c|}{ Total } & 902 \\
\hline
\end{tabular}

Os resultados do código de árvore do software webQDA ${ }^{\circledR}$, relativos aos fatores de risco, permite-nos constatar que existem 724 referências relativas à categoria mecânica corporal, 98 referências à ergonomia no bloco de partos e apenas 65 referências à atividade/tarefa.

Na subcategoria alinhamento corporal foi possível verificar que, das 184 referências, 125 mantinham alinhamento, e 59 não mantinham o alinhamento, visível nesta citação: "cabeça em flexão lateral direita, desalinhada em relação à coluna vertebral, ombros em elevação com inclinação lateral direita do tronco, coluna desalinhada".

Relativamente à subcategoria equilíbrio, detentora de 189 referências, das quais 130 faziam referência à adoção de equilíbrio e 59 ao desequilíbrio. Numa análise mais cuidada, verificamos que, os partos realizados na posição sentado, mantiveram o equilíbrio (base de sustentação, centro de gravidade e alinhamento) em todas as atividades, ao passo que, os partos realizados na posição ortostática, apresentaram maior desequilíbrio ao nível da manutenção da linha e centro da gravidade no que concerne às atividades proteção do períneo e libertação do ombro anterior. Relativamente à categoria fatores de risco organizacionais e psicossociais, constatou-se que a maioria das referências assenta na condução do parto por outro profissional (11 referências), como expresso na citação: "os dois médicos que se encontram na sala, dão orientações em simultâneo à grávida, tendo o mais antigo dominado a condução do parto", e apenas quatro faz referência à autonomia do enfermeiro obstetra na condução e realização do parto. 
Na observação mais cuidada dos vídeos, constatou-se que só se verificou desalinhamento corporal do enfermeiro obstetra que realiza o parto sentado, na orientação de outro profissional, constatado pelo desnivelamento dos ombros causado pela abordagem lateral ao períneo, consequência da existência de dois profissionais no campo de ação.

\section{DISCUSSÃO}

Relativamente aos princípios da biomecânica adotados pelo enfermeiro obstetra na assistência à parturiente, durante o $2^{\circ}$ e $3^{\circ}$ estádio em partos horizontais, verificamos que, devido à natureza da atividade profissional, estes passam longos períodos de pé, adotando posições corporais desalinhadas como a flexão da cabeça, o desalinhamento da coluna vertebral, a flexão e desalinhamento do tronco, a abdução e flexão dos membros superiores e a elevação constante dos ombros, indo ao encontro com outros resultados evidenciados por outros autores (Presado, Cardoso, Marques, \& Baixinho, 2017; Ganer, 2016; Baixinho et al., 2016a).

No segundo estádio, a proteção do períneo foi a atividade em que os EO não cumpriram os princípios da biomecânica, manifestado no desalinhamento e postura incorreta na posição ortostática. De salientar que esta atividade obteve elevado risco para LMELT segundo a avaliação REBA $(9,7)$, necessitando de uma intervenção de correção postural em breve. Segundo vários autores, as posturas forçadas ou estáticas por longos períodos, os movimentos repetitivos, e seus fatores de stress associados, coadunam-se com LMELT (Okuyucu, Jeve \& Doshani, 2017; Prezado et al., 2015;).

Constatou-se que, nos momentos complicados onde o stress está patente (circulares cervicais apertadas, bradicardia fetal, recém-nascido hipotónico e distocia de ombros), os enfermeiros obstetras adotam posturas onde o alinhamento corporal e o equilíbrio não são tidos em consideração, conclusão defendida por outros investigadores, salientando que, a elevada exigência das tarefas, os elevados níveis de pressão e stress, levam o sujeito a adotar posturas incorretas, aumentando o risco das LMELT (Yan et al., 2018).

Relativamente ao terceiro estádio, constatou-se que a colheita de sangue do cordão para células estaminais, foi a atividade onde a posição estática do EO é mais longa (duração média de 4 minutos). Posições estáticas mantidas por um longo tempo são, por si, um fator de risco de lesão que pode ser acrescida por outros riscos (Yan et al., 2018). 
O equilíbrio, que alguns autores referem ser o alicerce da biomecânica, sem o qual os restantes ficam comprometidos (Presado et al., 2017; Potter, Perry, Storkert \& Hall, 2013), foi a subcategoria da mecânica corporal que obteve maior número de unidades de enumeração e um dos princípios não assegurados no decorrer das atividades do enfermeiros obstetras, evidente na proteção do períneo.

Verificou-se que a força aplicada no momento da libertação dos ombros, também designada por carga, é muitas vezes realizada sem recurso ao peso corporal, obrigando a uma carga na apresentação cefálica do recém-nascido para conseguir a libertação do ombro anterior. Embora na perspetiva ergonómica existam muitos tipos de força que afetam a estrutura biomecânica, um desequilíbrio biomecânico acontece quando, a força exercida requer mais do que a capacidade da pessoa, ocorrendo um desequilíbrio que tem alto potencial para resultar em lesão (Okuyucu et al., 2017).

A especificidade das tarefas desenvolvidas no período expulsivo, estão associadas a riscos específicos, os movimentos bruscos de prono-supinação, na libertação do ombro posterior do recém-nascido, realizados com elevação das mãos em relação aos ombros (estes em tensão e elevação), a componente emocional e o stress do momento, são achados que vão ao encontro de outros estudos (Mineiro, Presado e Cardoso, 2019; Presado et al., 2017; Taghinejad, Azadi, Suhrabi \& Sayedinia, 2016).

$\mathrm{Na}$ análise dos vídeos e das opções adotadas pelos diferentes profissionais, na realização das atividades durante o segundo e terceiro estádio do trabalho de parto, concluímos que os profissionais que realizam o parto na posição sentada mantiveram o equilíbrio (base de sustentação, centro de gravidade e alinhamento) em todas as atividades, contrariamente ao verificado nos partos em posição ortostática, que apresentaram maior desequilíbrio ao nível da manutenção da linha e centro da gravidade, especificamente na proteção do períneo, libertação do ombro anterior e posterior do recém nascido, e na tração do cordão com ligeira pressão no fundo uterino. Segundo a OE, devem ser adotadas posturas onde o alinhamento corporal e o equilíbrio são assegurados, porque correspondem a uma correta mecânica corporal, minimizando os riscos de LMELT (OE, 2013).

Os profissionais de saúde que trabalham nos serviços de obstetrícia, apresentam longos períodos em posições ortostáticas, estáticas, de cócoras, inclinando-se, dobrando-se e em constante flexão do tronco, causando pressão no pescoço, ombros e tronco, expondo-os a LME (Mineiro et al, 2019; Wang et al., 2017). 
A antecipação dos problemas/consequências que ocorrem na prática clínica, é um aspeto fundamental na prevenção das LMELT, com a identificação das atividades que necessitam de intervenção imediata e em curto prazo (11 atividades), devendo-se proceder à intervenção multidimensional (prevenção, educação e formação dos profissionais), que segundo alguns autores, são mais efetivas relativamente à prevenção secundária e terciária (Hoof et al.,2018;Jerónimo, 2013).

A estimulação do períneo na região posterior e lateral, obteve um alto risco para LME, mas segundo as novas guidelines da WHO (2018), estas práticas não são recomendadas; no entanto, preconiza-se a massagem, a aplicação de compressas quentes e a proteção manual do períneo para evitar traumatismo, e a tração controlada do cordão umbilical (WHO,2018).

A abordagem qualitativa das LMELT nos profissionais de saúde é limitada, existindo apenas alguns estudos que focam o problema, através da sua visão qualitativa (Mineiro et al, 2019; Presado et al., 2017; Baixinho et al., 2016b; Ganer, 2016; Long, Bogossian \& Johnston, 2013).

A perda de autonomia do enfermeiro obstetra, visível na análise de alguns vídeos, provoca no profissional um sentimento de impotência, de inferioridade, de descontrolo da situação, que associado a outros fatores, aumentam o stress e o cansaço mental. Segundo Wang, et al. (2017), os fatores de stress psicológico no trabalho, podem exercer respostas fisiológicas (aumento e prolongar da tensão), que favorecem as LMELT.

$\mathrm{Na}$ génese das lesões podem estar atividades forçadas durante o trabalho de parto, como empurrar, puxar, e movimentação da parturiente ou objetos pesados. Estas atividades e as posturas corporais extremas, desconfortáveis e stressantes, estão muitas vezes associadas à não colaboração das parturientes, evidente em alguns estudos e no decorrer deste estudo (Okuyucu et al., 2017).

\section{CONCLUSÕES}

É imprescindível que o enfermeiro obstetra reflita sobre a sua prática clínica, analisando não só os cuidados prestados, mas também a biomecânica no decorrer da sua execução. Os princípios da ergonomia devem estar presentes na prática clínica, conduzindo a um sistema 
de cuidados produtivo, seguro, eficiente e de qualidade, tanto para os profissionais como para os utentes.

O desenvolvimento de estudos na área das LMELT, implica uma avaliação criteriosa e rigorosa das LMELT, pois só assim a enfermagem pode ser reconhecida como profissão de risco, com as inerentes doenças profissionais subjacentes.

Estes resultados orientam para a formação pós-graduada e contínua dos profissionais, auxiliando na identificação das posturas a intervir, e com o recurso à prática simulada de alta-fidelidade, procedendo à correção das mesmas. A investigação só tem sentido quando podemos fazer esta translação do conhecimento para a prática clínica, melhorando e desenvolvendo a sua práxis. (Baixinho et al., 2017; Nkhata, Louw, Brink, \& Mweshi, 2016).

A etiologia multifatorial das LMELT, torna este processo investigativo complexo e difícil. O rigor da seleção do método, seleção dos instrumentos de medida/colheita de dados, o recurso a peritos na área e a clarificação de toda a metodologia de investigação, são aspetos que conferem credibilidade ao trabalho desenvolvido. A potencialidade que 0 recurso aos vídeos tem, ficou bem patente neste trabalho, possibilitando a análise do fenómeno através do método misto (qualitativa e quantitativa), enriquecendo a análise dos resultados. Concordamos que a possibilidade do distanciamento emotivo para a análise reflexiva do material, com a possibilidade de rever ou congelar a imagem, são aspetos enriquecedores na análise dos fenómenos. O método qualitativo, possibilitou o estudo mais profundo do fenómeno, constatando outros fatores que intervêm nas $L M E L T$, que não são valorizados nos métodos quantitativos.

\section{REFERÊNCIAS}

Agência Europeia para a Segurança e Saúde no Trabalho (2007). Introdução às lesões músculo-esqueléticas. Belgium: FACTS 71. Acedido a 13/12/2017. Disponível em: http://osha.europa.eu

Akbari, H, Akbai, H., Abadi, M, Fesharaki, M. \& Ghaseni, M. (2016). Assessing the Risk of Manual Handling of Patients and Its relations with the prevalence of Musculoskeletal disorders among nursing staff: performance evaluation of the MAPO and PTAI methods. Iranian Red Crescent Medical Journal, 19 (2), 1-8.

Baixinho, C.L., Presado, H., Marques, F.M. \& Cardoso, M. (2016a). A segurança biomecânica na prática clínica dos enfermeiros de saúde materna e obstetrícia. Revista Brasileira Promoção da Saúde. Fortaleza, 29(sup), 3643

Baixinho C.L., Presado, H., Marques, F.M. \& Cardoso, M. (2016b). Prevenção de lesões músculo-esqueléticas: relatos dos enfermeiros especialistas em saúde materna e obstetrícia. Atas - Investigação Qualitativa em Saúde, 2, 488-497.

Baixinho, C.L., Ferreira, Ó., Marques, F.M., Presado, M.H. \& Cardoso, M. (2017). Transição segura: um projeto da 
transferência do conhecimento para a prática clínica. In A.P.Costa, M.C. Sánches-Gómez., M.V.M. Cilleros (Eds). A prática na Investigação Qualitativa: exemplos de estudos (57-80). Oliveira de Azeméis: Ludomédia

Bardin, Laurence (2013). Análise de conteúdo (reimpressão da edição revista e atualizada de 2009). Lisboa: Edição 70

Chung, Hung, Lee, Wang, Chang, Lee-Wen, Huang \& Yang (2013). Risk of musculoskeletal disorder among taiwanese nurses cohort: a nationwide population-based study.Biomedcentral.14(144),1-6

Costa, R., Grilo, A., Lemos, S., Silva, R., Silvestre, S., Marques, F. ... Baixinho, C. (2011). Lesões Músculoesqueléticas: Postura correta na escola, postura adquirida para a vida!. In P. Arezes, J. Baptista, M. Barroso, P. Carneiro, P. Cordeiro, N. Costa, ... G. Perestrelo (editores). Segurança e Higiene Ocupacionais - SHO 2011. (pp. 216-219). Guimarães: Universidade do Minho.

Davis, K, \& Kotowcki, S. (2015). Prevalence of Musculoskeletal Disorders for Nurses in Hospitals. Human Factors and Ergonomics Society. 57(5), 754-792.

Ellapen, T. \& Narsigan, S. (2014). Work related musculoskeletal disorders among nurses: systematic review. Journal of Ergonomics. S14:S14-003. 1-6 Acedido a 13/05/2018 Disponível em: https://www.omicsonline.org/open-access/work-related-musculoskeletal-disorders-among-nurses-systematicreview-2165-7556.S4-003.php?aid=26004

Fatia, A. \& Tinoco, L. (2016). Fisiologia do Trabalho de Parto. In M. Néné, R. Marques, \& M. Batista, Enfermagem de Saúde Materna e Obstétrica (pp. 321-323). Lisboa: Lidel

Flick U. (2004). Introducción a la investigación cualitativa. Madrid:Morata.

Ganer N. (2016). Work Related Musculoskeletal Disorders among Healthcare Professional and their Preventive Measure: a report. IJSRSET, 2(4), 693-698

Hignett, S. \& McAtamney, L. (2000). Rapid entire body assessment (REBA). Applied Ergonomics, 21, 201-205

Hoof, W., O’Sullivan, K., Keeffe, M., Verschueren, S., O’Sullivan, P. \& Dankaerts, W., (2018). The efficacy of interventions for low back pain in nurses A systematic. International Journal of Nursing studies, 77, $222-231$.

Jerónimo, J. (2013). Estudo da prevalência e fatores de risco de lesões musculoesqueléticas relacionadas com o trabalho em enfermeiros. Dissertação de Mestrado. Escola Superior de Enfermagem de Coimbra, Coimbra.

Lima, F. H. (2015). Um método de transcrição e análise de vídeos: a evolução de uma estratégia. In: VII Encontro Mineiro de educação matemática, Universidade Federal de São João del Rei, 9-12 outubro 2015 (pp.1-11).

Long, M., Bogossian, F., \& Johnston, V. (2013). The prevalence of work-related neck, shoulder, and upper back musculoskeletal disorders among midwives, nurses, and physicians. A systematic review. Workplace Health and Safety, 61(5), 223-229.

Mineiro A.L., Presado M.H. e Cardoso M. (2019).Posturas do Enfermeiro Obstetra na assistência ao parto em posições verticais. Investigação Qualitativa em Saúde//Investigación Cualitativa en Salud//Volume 2. 807816

Nkhata, L. A., Louw, Q., Brink, Y. \& Mweshi, M.M. (2016). Review on Effects of Ergonomic Interventions for Nurses on Function, NeuroMuscular Pain and Quality of Life. Journal of Preventive and Rehabilitative Medicine; 1(2), $53-60$.

Okuyucu, A., Jeve, Y., \& Doshani, A. (2017). Work-related musculoskeletal injuries amongst obstetrics and gynaecology trainees in East Midland region of the UK. Arch Gynecol Obstet, 296, 489-494.

Ordem dos enfermeiros (2013) Guia Orientador de boas práticas: Cuidados à pessoa com alterações da mobilidade-posicionamentos e transferências e treino de deambulação, 1(7), 1-77.

Potter, P.A., Perry, A.G., Storkert, P.A., \& Hall, A.M. (2013). Fundamentos de Enfermagem (8ª ed.). Rio de Janeiro: 
Elsevier Editora, Ltda.

Presado, M. H., Cardoso, M., Marques, F. M. \&. Baixinho, C.L. (2017). Posturas dos estudantes durante o trabalho

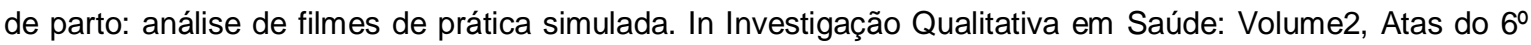
Congresso Ibero-Americano em Investigação Qualitativa (CIAIQ2017), 513-522

Prezado, M.H., Marques, F.M., Baixinho, C.L. \& Cardoso, M. (2015). Lesões músculo-esqueléticas nos enfermeiros especialistas em saúde materna: autoperceção dos fatores de risco. In Investigação Qualitativa em Saúde: Volume1, Atas do 4ํㅡㄹ Congresso Ibero-Americano em Investigação Qualitativa (CIAIQ2015), 5-7 agosto 2015 (pp.193-198)

Queiroz, M.V., Uva, A.S., Carnide, F., Serranheira, F., Miranda, L.C. \& Lopes, M.F. (2008). Lesões musculoesqueléticas relacionadas com o Trabalho - Guia de orientação para a prevenção: programa nacional contra as doenças reumáticas. Lisboa: Direção Geral de Saúde

Santos, A. R. V. (2015). Lesões musculosqueléticas relacionadas com o trabalho dos enfermeiros em contexto Hospitalar. Dissertação de Mestrado. Escola Superior de Enfermagem de Coimbra. Acedido a 13/12/2017 em Repositório Cientifico da ESEC. Disponível em: https://repositorio.esenfc.pt/rc/

Silva, A. \& Fossá, M. (2015). Analise de conteúdo: exemplo de aplicação da técnica para análise de dados qualitativos. Qualitas Revista eletrónica. 17(1).

Taghinejad, H., Azadi, A., Suhrabi, Z. \& Sayedinia, M. (2016). Musculoskeletal disorders and their related risk factors among Iranian nurses. Biotechnologyand Health Sciences. In Press. 10.17795/bhs-34473

Tsekoura, M, Koufogianni, A, Billis, E. \& Tsepis, E.(2017).Work-related musculoskeletal disorders among female and male nursing personnel in greece. WJRR. 3(1) 8-15

Wang, J, Cui, Y, Xu, X, Yuan, Z, Jin, X, \& Li, Z (2017). Work-Related Musculoskeletal Disorders and Risk Factors among Chinese Medical Staff of Obstetrics and Gynecology. International Journal of environmental research and public health. 14(562), 1-13

World Health Organization (2018). WHO recommendations: intrapartum care for a positive childbirth experience. Geneva: World Health Organization. Acedido a 25/2/2018. WHO Disponível em: http://apps.who.int/iris/bitstream/handle/10665/260178/9789241550215eng.pdf;jsessionid=7C81C5A9B5A4F1ED1AEC6EF1C2C4D83D? sequence $=1$

Yan, P, Yang, Y, Zhang, L., Li, F., Huang, A., Wang, Y., Dai, Y. \& Yao, H. (2018). Correlation analysis between work-related musculoskeletal disorders and the nursing practice environment, quality of life, and social support in the nursing professionals. Medicine, 97(9), 1-6 